\title{
BMJ Open Cost-effectiveness of ranibizumab and bevacizumab for age-related macular degeneration: 2-year findings from the IVAN randomised trial
}

\author{
Helen A Dakin, ${ }^{1}$ Sarah Wordsworth, ${ }^{1}$ Chris A Rogers, ${ }^{2}$ Giselle Abangma, ${ }^{3}$ \\ James Raftery, ${ }^{4}$ Simon P Harding, ${ }^{5}$ Andrew J Lotery, ${ }^{6}$ Susan M Downes, ${ }^{7}$ \\ Usha Chakravarthy, ${ }^{8}$ Barnaby C Reeves, ${ }^{2}$ on behalf of the IVAN Study Investigators
}

To cite: Dakin HA, Wordsworth S, Rogers CA, et al. Cost-effectiveness of ranibizumab and bevacizumab for agerelated macular degeneration: 2-year findings from the IVAN randomised trial. BMJ Open 2014:4:e005094.

doi:10.1136/bmjopen-2014005094

- Prepublication history and additional material is available. To view please visit the journal (http://dx.doi.org/ 10.1136/bmjopen-2014005094).

UC and BCR contributed equally.

Received 19 February 2014 Revised 19 June 2014 Accepted 23 June 2014

\section{CrossMark}

For numbered affiliations see end of article.

Correspondence to

Helen A Dakin;

helen.dakin@dph.ox.ac.uk

\section{ABSTRACT}

Objective: To assess the incremental cost and costeffectiveness of continuous and discontinuous regimens of bevacizumab (Avastin) and ranibizumab (Lucentis) for neovascular age-related macular degeneration (nAMD) from a UK National Health Service (NHS) perspective.

Design: A within-trial cost-utility analysis with a 2-year time horizon, based on a multicentre factorial, noninferiority randomised controlled trial.

Setting: 23 hospital ophthalmology clinics.

Participants: 610 patients aged $\geq 50$ years with untreated nAMD in the study eye.

Interventions: $0.5 \mathrm{mg}$ ranibizumab or $1.25 \mathrm{mg}$ bevacizumab given continuously (monthly) or discontinuously (as-needed) for 2 years.

Main outcome measures: Quality-adjusted life-years (QALYS).

Results: Total 2-year costs ranged from £3002/patient (\$4700; 95\% Cl £2601 to £3403) for discontinuous bevacizumab to £18 590/patient ( $\$ 29106 ; 95 \% \mathrm{Cl}$ $£ 18258$ to £18 922) for continuous ranibizumab. Ranibizumab was significantly more costly than bevacizumab for both continuous ( $+£ 14$ 989/patient ( $\$ 23468$ ); 95\% Cl £14 522 to $£ 15$ 456; $p<0.001$ ) and discontinuous treatment ( $+£ 8498$ ( $\$ 13305) ; 95 \% \mathrm{Cl}$ $£ 7700$ to $£ 9295 ; p<0.001$ ), with negligible difference in QALYs. Continuous ranibizumab would only be costeffective compared with continuous bevacizumab if the NHS were willing to pay £3.5 million (\$5.5 million) per additional QALY gained. Patients receiving continuous bevacizumab accrued higher total costs (+£599 (\$938); $95 \% \mathrm{Cl} £ 91$ to $£ 1107 ; p=0.021$ ) than those receiving discontinuous bevacizumab, but also accrued nonsignificantly more QALYs $(+0.020 ; 95 \% \mathrm{Cl}-0.032$ to $0.071 ; p=0.452)$. Continuous bevacizumab therefore cost $£ 30220$ ( $\$ 47316$ ) per QALY gained versus discontinuous bevacizumab. However, bootstrapping demonstrated that if the NHS is willing to pay $£ 20000 /$ QALY gained, there is a $37 \%$ chance that continuous bevacizumab is cost-effective versus discontinuous bevacizumab.

Conclusions: Ranibizumab is not cost-effective compared with bevacizumab, being substantially more

\section{Strengths and limitations of this study}

- We conducted a trial-based economic evaluation based on high-quality data on costs and quality of life prospectively collected within a randomised trial.

- This demonstrated that bevacizumab would achieve substantial cost-savings over ranibizumab with negligible differences in quality of life. In England, switching patients to bevacizumab could save at least $£ 102$ (\$160) million per year. However, bevacizumab is not currently licensed for neovascular age-related macular degeneration (nAMD).

- Our study is the first trial-based economic evaluation to evaluate the cost-effectiveness of alternative vascular endothelial growth factor inhibitor treatments for $\mathrm{nAMD}$.

- Of the strategies for the treatment of nAMD evaluated in this trial, we found discontinuous (as-needed) bevacizumab to be the least costly and most cost-effective. However, there was substantial uncertainty around this finding and sensitivity analyses suggested that the cost-effectiveness of using continuous (monthly) treatment rather than discontinuous treatment may vary between centres.

costly and producing little or no QALY gain.

Discontinuous bevacizumab is likely to be the most cost-effective of the four treatment strategies evaluated in this UK trial, although there is a $37 \%$ chance that continuous bevacizumab is cost-effective.

Trial registration number: ISRCTN92166560.

\section{INTRODUCTION}

Neovascular age-related macular degeneration (nAMD) is a common disorder of the ageing eye, which if left untreated leads to severe central visual impairment. The 
current standard of care is treatment with biologicals that bind to or inhibit vascular endothelial growth factor (VEGF). Biologicals need to be injected into the vitreous cavity of the eye at 4-8-week intervals. However, the first treatment convincingly shown to be effective in preventing vision loss (ranibizumab, Lucentis ${ }^{1}{ }^{2}$ ) is expensive $\left(£ 742 /\right.$ dose in the $\left.\mathrm{UK}^{3}\right)$. Another anti-VEGF biological, bevacizumab (Avastin), is licensed to treat cancer and has been used to treat nAMD, using smaller doses that cost much less than ranibizumab. Small non-randomised studies on bevacizumab have reported outcomes that were as good as those achievable with ranibizumab. ${ }^{4}$ Comparative effectiveness randomised controlled trials (RCTs) of ranibizumab versus bevacizumab were therefore needed to provide unbiased estimates of relative efficacy and safety. The UK Inhibition of VEGF in Age-related choroidal Neovascularisation (IVAN) trial $^{56}$ and the US Comparison of Age-related macular degeneration Treatments Trials (CATT) ${ }^{78}$ were among the first such trials to report findings.

Two-year IVAN results demonstrated that ranibizumab and bevacizumab produced similar improvements in visual function, with no significant difference in arteriothrombotic events or hospital admissions for heart failure, which have previously been linked with anti-VEGF therapy. ${ }^{5}$ IVAN also compared discontinuous (as-needed) treatment against continuous monthly injections. Continuous and discontinuous treatment produced similar improvements in visual function, although mortality was significantly lower with continuous treatment $(\mathrm{p}=0.05)$.

Given the rising demands for healthcare and limited budgets, it is important to assess cost-effectiveness as well as the clinical effectiveness and safety of medical interventions. Evidence on incremental cost and costeffectiveness is of particular importance in nAMD, owing to the potential savings and health implications of either reducing treatment frequency or substituting a much cheaper alternative (bevacizumab) for a more expensive drug (ranibizumab). Although ranibizumab costs many times more than bevacizumab, it is important to consider all relevant costs and assess cost-effectiveness to determine whether the more expensive therapy has added health benefits that justify the additional costs or lead to savings that offset the price difference.

A recent systematic review ${ }^{9}$ identified nine economic evaluations of ranibizumab and three of bevacizumab. Seven further studies evaluating ranibizumab ${ }^{10-16}$ and two studies evaluating bevacizumab ${ }^{11}{ }^{16}$ have since been published. Most studies found ranibizumab to be costeffective versus other treatments, such as pegaptanib. Five studies concluded that bevacizumab was likely to be cost-effective compared with ranibizumab, of which four studies relied on observational data ${ }^{11}{ }^{15}$ or assumptions about relative efficacy. ${ }^{17}{ }^{18}$ We are unaware of any other RCT-based economic evaluation that has estimated the cost-effectiveness of anti-VEGF treatment for nAMD.
A key objective of the IVAN trial was to assess the incremental cost and incremental cost-effectiveness of continuous and discontinuous regimens of bevacizumab and ranibizumab in nAMD from the perspective of the UK National Health Service (NHS). The results of these analyses are reported here.

\section{METHODS}

The study was based on the 2-year results from the IVAN trial (ISRCTN92166560), which provided high-quality data on resource use and outcomes and comprises the only UK trial directly comparing these interventions. Trial design and methods have been described previously $^{5}{ }^{6}$; in brief, this was a factorial, multicentre noninferiority trial in which 610 patients not previously treated for nAMD in their study eyes were randomised to either bevacizumab $(1.25 \mathrm{mg} /$ dose $)$ or ranibizumab $(0.5 \mathrm{mg} /$ dose $)$ and to either discontinuous treatment or continuous monthly injections for 2 years. Discontinuous treatment comprised an initial course of three monthly injections, followed by further courses of three injections given monthly if prespecified clinical and optical coherence tomography (OCT) retreatment criteria were met. The economic evaluation took a 2-year time horizon to estimate within-trial cost-effectiveness as incremental costs and quality-adjusted life-years (QALYs) appeared to be relatively stable over time. Following UK guidelines, ${ }^{19}$ we took the perspective of the UK NHS, which excludes costs incurred by patients and their families or employers. Detailed methods and additional results will be published as a monograph in Health Technology Assessment.

As IVAN was factorial, it was important to consider the likelihood of interactions, that is, whether the differences in costs and/or quality of life between bevacizumab and ranibizumab differ between treatment regimens. Although no interactions were anticipated for visual acuity, ${ }^{6}$ large interactions between drug and treatment regimen were expected for costs and costeffectiveness, as reducing the number of injections would have a proportionately greater effect on drug costs for ranibizumab than for less expensive bevacizumab. Interactions for quality of life or costs were also possible if the number of injections required for discontinuous treatment differed between drugs. We therefore estimated the mean costs and mean QALYs for each of the four treatment combinations and interpreted the results based on four pairwise comparisons:

- Continuous ranibizumab versus discontinuous ranibizumab,

- Continuous bevacizumab versus discontinuous bevacizumab,

- Continuous ranibizumab versus continuous bevacizumab,

- Discontinuous ranibizumab versus discontinuous bevacizumab.

We report two forms of economic evaluation. Comparisons between drugs were based on cost-minimisation 
analysis, which compares costs between treatments that are assumed to have identical health effects. ${ }^{20}$ Cost-minimisation analysis is appropriate only if the difference in cost is so large that no plausible difference in efficacy could cause the more costly treatment to be costeffective. ${ }^{21} 22$ This approach is justified for the comparisons between drugs because the large difference in drug costs was inevitably going to be the main influence on the incremental cost-effectiveness of ranibizumab versus bevacizumab. We therefore prespecified that ranibizumab and bevacizumab would be compared using costminimisation analysis unless ranibizumab-treated patients accrued $\geq 0.05$ more EQ-5D QALYs than those receiving bevacizumab. In contrast, we used cost-utility analysis, in which health outcomes are measured in QALYs, to compare continuous and discontinuous treatment, where incremental costs are smaller.

\section{Measurement and valuation of resource use}

Our base case analysis also focused on resource use associated with the study eye or associated with adverse events (AEs) or serious adverse events (SAEs) that were 'expected', that is, previously linked to anti-VEGF treatment (see online supplementary appendix). Concomitant medications, hospitalisations and ambulatory consultations that were neither associated with the study eye nor attributable to expected AEs or expected SAEs were excluded to avoid including episodes of high healthcare resource use unrelated to treatment (eg, renal failure or cancer), which might otherwise have swamped the main effect of treatment on costs. ${ }^{23}$

After enrolment, participants were monitored for disease activity on a monthly basis with visual acuity assessments, colour fundus imaging and OCT. Fundus fluorescein angiography (FFA) was undertaken at specified visits and when OCT was insufficient to reach a decision on disease activity. A prespecified algorithm was used to determine the need for retreatment. Patients allocated to discontinuous treatment began a new course of three monthly injections whenever they met retreatment criteria. However, costing analyses excluded protocol-driven resource use; in particular, we assumed that patients would not require colour fundus photography, OCT or FFA unless this would affect treatment decisions. As such, patients on discontinuous treatment were assumed not to require these investigations at the second or third visit in a course of three injections, when treatment was mandated (figure 1). Similarly, patients on continuous treatment were assumed to require monitoring consultations only once every 3 months, on the grounds that ophthalmologists would want information about disease progression periodically, irrespective of whether treatment decisions are required.

Microcosting was used to estimate the cost of injection and monitoring consultations as the available national average $\operatorname{costs}^{24} 25$ are not nAMD-specific and do not differentiate between consultations for monitoring and intravitreal drug delivery. Staff at 13 of the 23 IVAN centres completed questionnaires on overheads, the cost of setting up clinic facilities and equipment and/or the staff required to run injection and monitoring clinics.

The drug acquisition cost for ranibizumab was the NHS list price $\left(£ 742.17^{3}\right)$ and that for bevacizumab was the price typically charged by the not-for-profit NHS provider used in the trial ( $£ 49 /$ prefilled syringe). All concomitant medications, contacts with medical professionals and hospitalisations were recorded at each monthly clinic visit. Concomitant medications applied to the study eye or indicated for any expected SAE/AE were valued using list prices. ${ }^{3}$ Costs of other medications, including those applied to the fellow eye, were excluded from the analysis. Unit costs for consultations with general practitioners, district or general practice nurses and hospital staff outside IVAN clinics were obtained from routine sources. ${ }^{25} 26$ These costs were applied to ambulatory consultations that were either related to the eye or that occurred within 30 days of an expected SAE/AE. Hospital stays linked to expected

\begin{tabular}{|l|l|l|l|l|l|l|l|l|l|l|l|l|l|}
\hline & Visit & $\mathbf{0}$ & $\mathbf{1}$ & $\mathbf{2}$ & $\mathbf{3}$ & $\mathbf{4}$ & $\mathbf{5}$ & $\mathbf{6}$ & $\mathbf{7}$ & $\mathbf{8}$ & $\mathbf{9}$ & $\mathbf{1 0}$ & $\mathbf{1 1}$ \\
\hline \multirow{3}{*}{$\begin{array}{l}\text { Patient 1: } \\
\begin{array}{l}\text { Continuous } \\
\text { treatment }\end{array}\end{array}$} & Injection & $\checkmark$ & $\checkmark$ & $\checkmark$ & $\checkmark$ & $\checkmark$ & $\checkmark$ & X & $\checkmark$ & $\checkmark$ & $\checkmark$ & $\checkmark$ & $\checkmark$ \\
\hline & $\begin{array}{l}\text { Monitoring } \\
\text { consult }\end{array}$ & $\checkmark$ & & & $\checkmark$ & & \multicolumn{10}{|c|}{ X } & & & $\checkmark$ & & \\
\hline & FFA & $\checkmark$ & & & $?$ & & \multicolumn{10}{|c|}{ X } & & & $?$ & & \\
\hline \multirow{2}{*}{$\begin{array}{l}\text { Patient 2: } \\
\text { Discontinuous } \\
\text { treatment }\end{array}$} & Injection & $\checkmark$ & $\checkmark$ & $\checkmark$ & & & & & $\checkmark$ & $\checkmark$ & $\checkmark$ & & $\checkmark$ \\
\hline & $\begin{array}{l}\text { Monitoring } \\
\text { consult }\end{array}$ & $\checkmark$ & & & $\checkmark$ & $\checkmark$ & $X$ & $\checkmark$ & $\checkmark$ & & & $\checkmark$ & $\checkmark$ \\
\hline & FFA & $\checkmark$ & & & $?$ & $?$ & $X$ & $?$ & $?$ & & & $?$ & $?$ \\
\hline
\end{tabular}

Figure 1 Schematic illustrating the assumptions made about the frequency of injection and monitoring consultations within the costing analysis. The consultations required by patients on discontinuous treatment will depend on when they met treatment failure criteria; patient 2 met the retreatment criteria at visits 0,7 and 11. $\checkmark$ Relevant consultation cost was applied. ? The cost of fundus fluorescein angiography (FFA) was applied if clinically indicated: for discontinuous patients, this was applied whenever the patient had FFA in the trial; for continuous patients, the proportion of patients having FFA was based on estimated use in routine clinical practice. $\mathbf{X}$ No consultation cost was applied as the participant missed the visit. 
SAEs were valued using the mean cost per bed-day for associated healthcare resource groups (HRGs). ${ }^{25}$

Resource use data and unit costs were combined to estimate quarterly costs of bevacizumab/ranibizumab; drug administration and monitoring consultations; and hospitalisations, ambulatory consultations and medication changes for expected SAEs/AEs. Value added tax (VAT) was excluded from the economic evaluation and included in budget impact estimates, following guidelines. ${ }^{19}$ Costs are reported in 2011 pounds sterling, accompanied by equivalents in US dollars (exchange rate: $\$ 1.57$ /pound).

\section{Measurement and valuation of health benefits}

The three-level EQ-5D questionnaire ${ }^{27}$ was administered at baseline, 3, 12 and 24 months, and (if the patient was willing and able to do so) at study exit, after any SAE and after a drop in visual acuity in the study eye of $\geq 15$ letters on the Early Treatment Diabetic Retinopathy Study vision chart between two consecutive visits (referred to subsequently as a 'reduction in visual acuity'). The Health Utilities Index questionnaire Mark 3 (HUI3) was administered at the same timepoints and used in sensitivity analysis; EQ-5D comprised the primary utility measure following UK guidelines. ${ }^{19}$ Patients selfcompleted large-print EQ-5D questionnaires, with assistance from study nurses where necessary, responses were valued using the UK time-trade-off tariff to give 'utilities'. ${ }^{27}$

Missing utility data were imputed using multiple imputation, ${ }^{28}$ which avoids bias and enables analysis of the whole sample. Multiple imputation was conducted using the ice command ${ }^{29}$ (V.1.9.4) in Stata V.12 (StataCorp, College Station, Texas, USA).

QALYs for each participant were calculated as the area under the curve. We assumed that utility changed linearly between consecutive EQ-5D measurements in the absence of SAEs. As linear changes are unlikely for patients with SAEs, we assumed that SAEs and reductions in visual acuity caused a sudden drop in utility on the day of onset, followed by a linear rise as the patient recovered; the rate of this linear rise was estimated using mixed models (see online supplementary appendix).

\section{Statistical methods}

Linear regression models were used to estimate the effect of drug and treatment regimen on QALYs, drug costs, administration/monitoring costs and medication/ medical service use in each 3-month period or 'quarter' (see online supplementary appendix). Interactions between drug and treatment regimen were included in the models for quarters 2-8 if they were either statistically significant or were larger than the main effect for drug or for treatment regimen. The analysis of QALYs, drug costs and medication/medical service use in quarters 2-8 therefore took account of interactions, while drug and treatment regimen were assumed to have additive effects on administration/monitoring costs.
A variant of Kaplan-Meier sample averaging ${ }^{30} 31$ was used to account for patients withdrawing early from the trial and excludes differences in mortality unrelated to treatment; regression predictions of quarterly costs and QALYs were weighted by the proportion of patients alive at the start of each quarter. Costs and QALYs accrued in year 2 were discounted at $3.5 \%$ to allow for time preference (ie, the tendency to prefer benefits sooner and costs later).$^{19}$ Uncertainty around quarterly costs and QALYs was quantified by estimating models separately for 130 non-parametric bootstrap draws on each of 100 data sets generated in multiple imputation to capture the uncertainty around imputed utilities. The appendix gives further details of the statistical methodology.

\section{Presentation of results and uncertainty}

Incremental cost-effectiveness ratios (ICERs) were calculated by dividing the difference in cost between two study arms by the difference in QALYs. Results were interpreted assuming that the UK NHS would be willing to pay $£ 20000$ to gain one QALY (a £20 000/QALY 'ceiling ratio'). ${ }^{32}$ We also present net benefits for each of the four treatment arms: net benefit equals total QALYs multiplied by the ceiling ratio, minus total costs. Uncertainty around ICERs is presented as costeffectiveness acceptability curves, which plot the probability of each of the four treatment regimens having the highest net benefits (ie, being most cost-effective) at a range of ceiling ratios.

Sensitivity analyses evaluated the impact of changing the costs (eg, halving the cost of ranibizumab), methods (eg, taking a 1-year time horizon) and assumptions (eg, including the costs of all SAEs, not just 'expected' SAEs).

\section{RESULTS}

\section{QALYs and quality of life}

The number of QALYs accrued over the 2-year trial period did not differ significantly between bevacizumab and ranibizumab, or between continuous and discontinuous treatments $(\mathrm{p} \geq 0.381$; table 1$)$. Patients randomised to continuous treatment accrued non-significantly more QALYs than those randomised to discontinuous treatment (mean difference: 0.020 (95\% CI -0.032 to 0.071 ) for bevacizumab, $\mathrm{p}=0.452$ and 0.026 (95\% CI -0.032 to 0.085 ) for ranibizumab, $\mathrm{p}=0.381$ ), while differences between ranibizumab and bevacizumab were negligible.

\section{Resource use and costs}

Patients receiving continuous treatment received a mean of 22 injections, while those on discontinuous treatment received 13 injections. Consequently, drug costs differed substantially between continuous and discontinuous treatments (table $1 ; \mathrm{p}<0.001$ ), as well as between ranibizumab and bevacizumab $(\mathrm{p}<0.001)$. As reducing treatment frequency produces larger savings for ranibizumab 


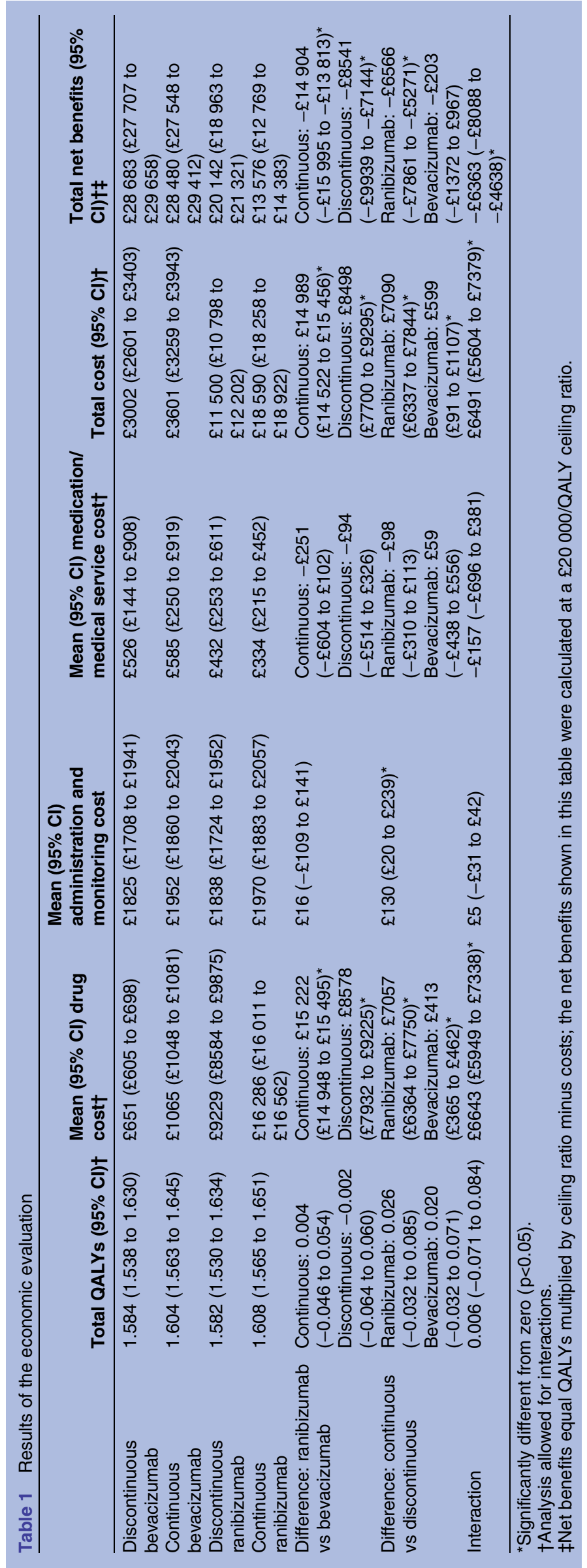

than for bevacizumab, there were significant interactions between drug and treatment regimen for drug cost $(\mathrm{p}<0.001)$.

Administration of bevacizumab or ranibizumab costs $£ 61$ (\$96; SD: £14) per injection, while each consultation for monitoring costs £72 (\$113; SD: £41), plus £39 (\$61; SD: £16) for each FFA. Administering intravitreal injections and monitoring disease progression/remission cost between $£ 1825$ and $£ 1970$ per patient over the 2-year trial period (table 1). Discontinuous treatment reduced the number of injections required, but increased the number of monitoring consultations needed to assess disease status against retreatment criteria, as we assumed that OCT would only be performed when it would inform treatment decisions. As continuous treatment requires, on average, nine more injections $(\mathrm{p}<0.001)$, but avoids only six monitoring visits $(\mathrm{p}<0.001)$, drug administration and monitoring costs were higher with continuous treatment than discontinuous treatment (mean difference: $£ 130$ per patient (\$204); 95\% CI $£ 20$ to $£ 239 ; \mathrm{p}=0.021$ ), with no significant difference between bevacizumab and ranibizumab $(\mathrm{p}=0.80)$.

The cost of medication changes, hospitalisations and ambulatory consultations associated with expected SAEs and expected AEs was relatively small (mean: $£ 469$ (\$735) per patient), but varied substantially between patients (95th centile range: £0, £1401). There was no significant difference in such costs between drugs or between treatment regimens $(p \geq 0.163)$.

Taking account of the drug cost, drug administration/ monitoring and medication/medical service use, the mean total cost per patient over the 2-year trial ranged from $£ 18590$ (\$29 119) for continuous ranibizumab to $£ 3002$ (\$4702) for discontinuous bevacizumab (table 1). Drug cost accounted for $80-88 \%$ of the total cost for patients randomised to ranibizumab and $21-30 \%$ of the cost for patients randomised to bevacizumab. Drug administration and monitoring accounted for 54-61\% of the costs accrued by patients randomised to bevacizumab and $10-15 \%$ of the costs for those randomised to ranibizumab.

\section{Base case comparison between ranibizumab and bevacizumab}

As the difference in mean QALYs between ranibizumab and bevacizumab was less than the prespecified noninferiority margin (0.05 QALYs), cost-minimisation analysis was used to compare the two drugs on the basis of cost alone. Overall, continuous ranibizumab cost $£ 14989$ more per patient ( $\$ 23476$ (95\% CI $£ 14522$ to $£ 15$ 456), table 1) than continuous bevacizumab over the 2-year trial period $(\mathrm{p}<0.001)$. Discontinuous ranibizumab costs $£ 8498$ more per patient ( $\$ 13308$ (95\% CI $£ 7700$ to $£ 9295$ ), $\mathrm{p}<0.001$ ) compared with discontinuous bevacizumab. Bootstrapping analyses estimated the probability that switching from ranibizumab to bevacizumab would save money and found that this exceeds $99.9 \%$. 
Base case comparison between continuous and discontinuous treatment

Overall, using continuous rather than discontinuous treatment increased costs by $£ 7090$ (\$11 102 (95\% CI $£ 6337$ to £7844), $\mathrm{p}<0.001$ ) for ranibizumab and $£ 599$ ( $\$ 938$ (95\% CI £91 to £1107), $\mathrm{p}=0.021$ ) for bevacizumab.

However, patients randomised to continuous bevacizumab also accrued non-significantly more QALYs than those randomised to discontinuous bevacizumab (table 1; $\mathrm{p}=0.452)$. In line with best practice, ${ }^{20}$ we took account of the non-significant differences in QALYs and allowed for the joint distribution of costs and QALYs, as assuming no difference in health outcomes can introduce bias and give misleading conclusions. ${ }^{21}{ }^{22}$ Dividing the difference in cost by the difference in QALYs suggests that continuous bevacizumab costs $£ 30220$ (\$47316) per additional QALY gained compared with discontinuous bevacizumab. This ICER is somewhat higher than the $£ 20000$ (\$31 000) per QALY 'ceiling ratio' below which the NHS generally considers treatments to be costeffective. ${ }^{32}$ However, the imprecision around QALY differences means that there is substantial uncertainty around this ICER. Bootstrapping demonstrated that there is a $37 \%$ chance that continuous bevacizumab is cost-effective compared with discontinuous bevacizumab at a $£ 20000 /$ QALY ceiling ratio, which increases to $50 \%$ at $£ 30000$ /QALY.

Continuous ranibizumab costs £270 217 (\$423 074) per QALY gained compared with discontinuous ranibizumab. Owing to the substantial savings possible by giving ranibizumab less frequently, we can be $>99.99 \%$ confident that continuous ranibizumab is poor value for money compared with discontinuous ranibizumab at a $£ 20$ 000/QALY ceiling ratio.

\section{Base case four-way comparison}

It is also informative to consider the four trial treatment groups as four mutually exclusive alternative strategies for managing nAMD. Framing the decision in this way demonstrates that discontinuous bevacizumab is the most cost-effective treatment strategy evaluated in IVAN, generating higher net benefits than the other three treatment strategies (table 1), where net benefit equals QALYs multiplied by ceiling ratio (in this case $£ 20000$ / QALY) minus costs. Continuous ranibizumab would only be cost-effective compared with continuous bevacizumab if the NHS were willing to pay $£ 3.5$ million ( $\$ 5.5$ million) per additional QALY gained. Discontinuous ranibizumab is not cost-effective at any ceiling ratio, as it is more costly and less effective than continuous or discontinuous bevacizumab.

However, there remains substantial uncertainty around incremental QALY gains. This is illustrated by the costeffectiveness acceptability curves plotting the probability of each treatment being the most cost-effective of the four strategies at different ceiling ratios (figure 2). This demonstrates that although we can be $98 \%$ confident that discontinuous bevacizumab is less costly than

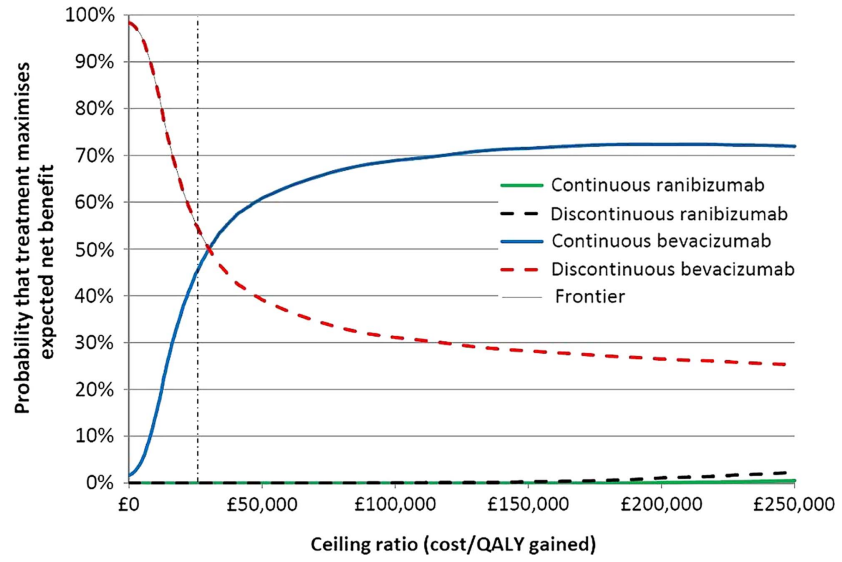

Figure 2 Cost-effectiveness acceptability curve showing the probability that each treatment is the most cost-effective strategy evaluated in the UK Inhibition of VEGF in Age-related choroidal Neovascularisation trial at a range of ceiling ratios. For example, at a ceiling ratio of $£ 20000$ /quality-adjusted life-year (QALY) gained (shown by the vertical dashed line), there is a $63 \%$ probability that discontinuous bevacizumab is best and a $37 \%$ probability that continuous bevacizumab is best, while the probability that either ranibizumab treatment regimen is best is approximately $0 \%$ (total $=100 \%$ ).

continuous bevacizumab, our confidence in the conclusion that discontinuous bevacizumab has the highest net benefits decreases rapidly as the value we place on the small, non-significant QALY gains increases. At a $£ 20000$ /QALY ceiling ratio, there is a $63 \%$ probability that discontinuous bevacizumab is the most cost-effective strategy considered in IVAN and a $37 \%$ probability that continuous bevacizumab is the most cost-effective. In contrast, the probability of either continuous or discontinuous ranibizumab being the most cost-effective strategy for managing nAMD is $<1 \%$ unless the NHS were willing to pay more than $£ 100000 / \mathrm{QALY}$ gained.

\section{Sensitivity analyses}

Sensitivity analyses demonstrated that the conclusions are very robust to changes in the assumptions and methods used to measure costs and utilities and conduct the analysis (figure 3). Of note, no sensitivity analysis changed the conclusion that ranibizumab is not costeffective compared with bevacizumab, including analyses discounting the ranibizumab list price by $50 \%$. However, three sensitivity analyses changed the conclusion that continuous bevacizumab is not cost-effective compared with discontinuous bevacizumab: assuming that FFA is only conducted at baseline, not at any subsequent monitoring consultation; measuring quality of life using HUI3 rather than EQ-5D; and using unadjusted Kaplan-Meier estimates of the probability of surviving at any point in time to account for censoring, rather than excluding differences in deaths that were unrelated to study medication (see online supplementary appendix).

Threshold analyses demonstrated that the price of ranibizumab would need to be reduced to $£ 63.46$ per 
Figure 3 Effect of sensitivity analyses on total net benefits for each of the four treatment arms, assuming a £20 000/ quality-adjusted life-year (QALY) ceiling ratio. Treatments that are more cost-effective have higher net benefits; the treatment furthest to the right is therefore most cost-effective, while the treatment furthest to the left is the least cost-effective. Error bars represent $95 \% \mathrm{Cls}$. In the analysis 'doubling SAE impact', both the medication/medical service use cost and the impact of serious adverse events (SAEs) on QALYs were doubled. The 'best case' analysis simultaneously changed several assumptions in favour of ranibizumab: $50 \%$ discount off the ranibizumab list price; assuming that $15.9 \%$ of bevacizumab (as occurred in the trial) but no ranibizumab is wasted; assuming that bevacizumab costs $£ 100$ per dose; and including medical expected and unexpected adverse events (AEs) and SAEs. service use costs associated with

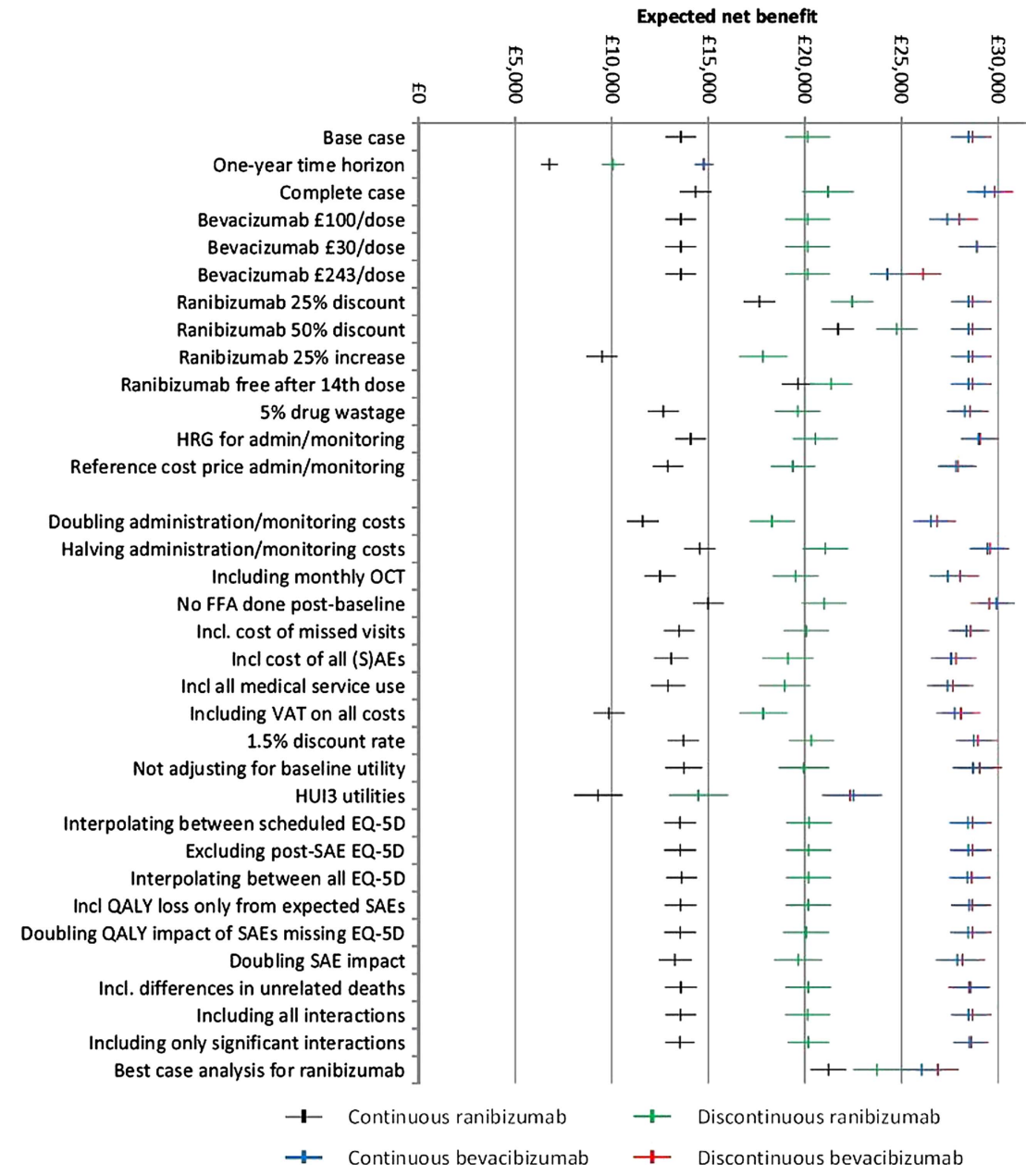

dose (a 91\% price reduction) in order for continuous ranibizumab to be cost-effective compared with continuous bevacizumab at a $£ 20000 /$ QALY ceiling ratio.

\section{DISCUSSION}

This study demonstrates that in the setting of the UK IVAN trial, we can be $>99 \%$ confident that ranibizumab represents very poor value for money compared with bevacizumab at the $£ 20000$ (\$31 000) per QALY ceiling ratio used within NHS decision-making. ${ }^{32}$ Continuous ranibizumab would only be cost-effective compared with continuous bevacizumab if the NHS were willing to pay $>£ 3.5$ (\$5.5) million/QALY gained. Furthermore, our analysis also shows that giving discontinuous bevacizumab, rather than discontinuous ranibizumab, could save the UK NHS $£ 8498$ (\$13 341) per patient treated, with little or no impact on the health gains from treatment. If the 17295 eyes requiring anti-VEGF therapy each year in England ${ }^{33}$ were switched from discontinuous ranibizumab to discontinuous bevacizumab, the NHS could save at least $£ 102$ (\$160) million per year (including $20 \%$
VAT) based on the treatment regimens evaluated in IVAN. It remains controversial as to whether a drug (bevacizumab) that has not been approved and licensed for nAMD by regulatory agencies should be used when a licensed drug (ranibizumab) is available. In the UK, clinicians may prescribe unlicensed medications within approved research projects, when no suitable medicine is licensed, or when the licensed alternative is unavailable, ${ }^{34}$ although prescribing on cost grounds is not mentioned. By contrast, in the USA, ophthalmologists use bevacizumab freely. ${ }^{35}$ National guidance (rather than local hospital/clinician policies) is therefore needed in the UK to direct the choice between bevacizumab and ranibizumab. CATT and IVAN provide robust data to guide regulators in this decision, demonstrating that ranibizumab and bevacizumab have comparable effects on vision and similar safety profiles, ${ }^{5}{ }^{7}$ but that (based on the current analysis of IVAN) ranibizumab costs $£ 3.5$ million per QALY compared with bevacizumab.

The base case analysis found that continuous bevacizumab costs $£ 30220$ (\$47 445) per QALY gained compared with discontinuous bevacizumab, suggesting that 
discontinuous bevacizumab is the most cost-effective strategy evaluated in IVAN if the NHS is willing to pay up to $£ 20000 /$ QALY gained. However, there remains substantial uncertainty around this conclusion and there is a $37 \%$ chance that continuous bevacizumab is costeffective. The finding of non-significantly higher QALYs with continuous treatment contradicts our prior hypothesis that avoiding monthly injections might improve quality of life, although the observed difference could be due to chance. Nevertheless, discontinuous bevacizumab would remain the most cost-effective strategy even if there were no difference in quality of life between treatment regimens. Other considerations may affect the choice of the anti-VEGF delivery model. In particular, as discontinuous treatment requires regular clinical review and access to retinal imaging, it may be more practical to provide treatment every month, with monitoring restricted to specified points in time (eg, 6 or 12 months after initiation of therapy). Indeed, the label for the newest anti-VEGF (aflibercept) incorporates a limited clinical monitoring regime. ${ }^{36}$ The discontinuous treatment regimen evaluated in the IVAN trial was chosen partly to minimise the possibility of disadvantage to participants in these groups and partly to minimise the number of retreatment decisions required. Neither monthly treatment nor treatments given as blocks of three are used widely in routine practice, although following the publication of IVAN, ${ }^{5}{ }^{6}$ there appears to be increased interest in the 'IVAN regimen'. The costeffectiveness of monthly treatment versus intermittent treatment will therefore vary between treatment centres depending on local costs and clinical practice.

Unlike previous studies, our analysis is based on highquality data from an RCT with prospective measurements of costs and quality of life, which was powered to exclude any clinically meaningful difference in visual acuity. It therefore provides unequivocally unbiased estimates of incremental costs and QALYs. Nevertheless, our analysis confirms the findings of previous economic evaluations, namely that ranibizumab is not cost-effective compared with bevacizumab. ${ }^{11} 1618$ We are also (to the best of our knowledge) the first to evaluate the costeffectiveness of the discontinuous treatment regimen used in IVAN. In addition to following best practice for trial-based economic evaluation, this study includes several novel aspects, such as measuring quality of life after SAEs, excluding chance differences in deaths unrelated to treatment and allowing for the factorial design by including only large or statistically significant interactions.

The study also estimates the cost of consultations to administer ranibizumab/bevacizumab and monitor outcomes, which could be used in other economic evaluations. Microcosting shows the main drivers of consultation costs and highlighted substantial variation in costs between centres; this variation means that the cost-effectiveness of continuous versus discontinuous bevacizumab (but not ranibizumab vs bevacizumab) will vary between centres. It is important to note that the costs were calculated to assess incremental costeffectiveness in IVAN and should not be used to set the prices at which hospitals are reimbursed. In particular, they are bottom-up estimates that exclude unpaid overtime and VAT and make assumptions about overheads and proportion of staff-time spent on patient contacts. In most settings, it is likely that the costs to healthcare commissioners will be higher and subject to local negotiations with care providers.

The base case analysis focused on mortality attributable to study medication and the costs associated with 'expected' SAEs/AEs and excluded other costs. This reduced the risk that chance differences in resource use not associated with study medication could distort our conclusions. However, it also meant that the unanticipated increase in the incidence of other SAEs (eg, gastrointestinal events) with bevacizumab ${ }^{5}{ }^{6}$ (which comprised the only difference in SAEs between drugs) was not taken into account in the costing analysis. However, sensitivity analyses including the cost of all SAEs/AEs gave the same conclusions. Although hospitals receive a commercial-inconfidence discount off the list price of ranibizumab and the price of bevacizumab varies between hospitals, the conclusions were robust to substantial changes in drug price. The study focused on the period of follow-up in the trial and excluded costs and benefits beyond year 2 . However, as incremental costs and QALYs remained reasonably stable over time, this is unlikely to have affected the conclusions. The analysis also uses data only from IVAN, rather than synthesising all available evidence.

Further research is needed to assess the extent to which the cost-effectiveness findings generalise to other countries with different relative prices and management of nAMD and SAEs/AEs. For example, the incidence of SAEs was substantially lower in IVAN than CATT, ${ }^{5} 7$ although sensitivity analyses doubling the impact of SAEs on costs and QALYs suggested that this did not change the conclusions. The costs of the two drugs may vary between centres within the UK as hospitals may use different bevacizumab suppliers or have different discounts on ranibizumab. Nevertheless, as we collected very detailed information on resource use, policymakers in other countries can review these data against their own to examine their similarity and hence the applicability of our findings to their setting. Future work combining data from IVAN with that from other trials, such as CATT, ${ }^{7}$ may help reduce uncertainty and evaluate the extent to which the results can be generalised. However, we believe that our primary finding of ranibizumab representing very poor value for money compared with bevacizumab does apply throughout the world.

Author affiliations

${ }^{1}$ Nuffield Department of Population Health, Health Economics Research Centre, University of Oxford, Oxford, UK

${ }^{2}$ Clinical Trials and Evaluation Unit, School of Clinical Sciences, University of Bristol, Bristol, UK

${ }^{3}$ Swiss Re Services Ltd, Research and Development, London, UK 
${ }^{4}$ Primary Care \& Population Sciences, University of Southampton,

Southampton, UK

${ }^{5}$ Department of Eye and Vision Science, Institute of Ageing and Chronic Disease, University of Liverpool, Liverpool, UK

${ }^{6}$ Academic Unit of Clinical and Experimental Sciences, Faculty of Medicine, University of Southampton, Southampton, UK

${ }^{7}$ Oxford Eye Hospital, Oxford University Hospitals NHS Trust, Oxford, UK ${ }^{8}$ Centre for Experimental Medicine, The Queen's University of Belfast, Belfast, UK

Acknowledgements The authors would like to thank the IVAN participants and the clinicians, nurses and clinic managers who completed the resource use questionnaires.

Collaborators The IVAN study investigators are listed online (available at http://aaojournal.org) in supplementary appendix 1 of ref. 6 .

Contributors SW, CAR, JR, SPH, AJL, SMD, BCR and UC conceived, designed and conducted the IVAN trial. HAD, SW, GA and JR conceived and designed the economic evaluation, with extensive input from CAR, SPH and BCR. CAR supervised the collation/cleaning of trial data, while HAD, SW and GA collected additional resource use data from centres. HAD conducted the economic analysis with help from GA under the supervision of SW. HAD drafted the manuscript. All authors edited the manuscript for important intellectual content and approved the final version.

Funding The IVAN trial is funded by the National Institute for Health Research (NIHR) Health Technology Assessment (HTA) programme (project number 07/36/01) and a full report will be published in Health Technology Assessment.

Competing interests All authors had financial support from the NIHR for the submitted work. UC, SH, SMD and AJL are principal investigators of trials sponsored by Novartis, the manufacturers of ranibizumab. UC has attended and been remunerated for attendance at advisory boards for Novartis, Bayer, Neovista, Oraya, Allergan, and Bausch and Lomb; and her employing institution has received payments from Novartis, Bayer, Neovista, Oraya, Alcon and Pfizer. SH has attended and been remunerated for attendance at advisory boards for and received travel support from Novartis and Allergan. CAR has received an honorarium from Novartis for a lecture. SMD's and AJL's employing institutions have received payments from Novartis. SMD and AJL have received honoraria from Novartis for lectures. AJL has attended and been remunerated for attendance at advisory boards for Novartis and Bayer; likewise, SMD has been remunerated by Bayer and Ely Lilly. BCR has received a fee for teaching from Janssen-Cilag.

Ethics approval A UK National Health Service Research Ethics Committee approved the trial (07/NIR03/37).

Provenance and peer review Not commissioned; externally peer reviewed.

Data sharing statement No additional data are available.

Open Access This is an Open Access article distributed in accordance with the Creative Commons Attribution Non Commercial (CC BY-NC 3.0) license, which permits others to distribute, remix, adapt, build upon this work noncommercially, and license their derivative works on different terms, provided the original work is properly cited and the use is non-commercial. See: http:// creativecommons.org/licenses/by-nc/3.0/

\section{REFERENCES}

1. Brown DM, Michels M, Kaiser PK, et al. Ranibizumab versus verteporfin photodynamic therapy for neovascular age-related macular degeneration: two-year results of the ANCHOR study. Ophthalmology 2009;116:57-65.e5.

2. Rosenfeld PJ, Brown DM, Heier JS, et al. Ranibizumab for neovascular age-related macular degeneration. $N$ Engl J Med 2006;355:1419-31.

3. British Medical Association. British National Formulary 62. London: Pharmaceutical Press, 2011. http://bnf.org/bnf/index.htm

4. Mitchell P. A systematic review of the efficacy and safety outcomes of anti-VEGF agents used for treating neovascular age-related macular degeneration: comparison of ranibizumab and bevacizumab. Curr Med Res Opin 2011;27:1465-75.

5. Chakravarthy U, Harding SP, Rogers CA, et al. Alternative treatments to inhibit VEGF in age-related choroidal neovascularisation: 2-year findings of the IVAN randomised controlled trial. Lancet 2013;382:1258-67.

6. Chakravarthy U, Harding SP, Rogers CA, et al; The IVAN Study Investigators. Ranibizumab versus bevacizumab to treat neovascular age-related macular degeneration: one-year findings from the IVAN randomized trial. Ophthalmology 2012;119:1399-411.

7. Martin DF, Maguire MG, Fine SL, et al. Ranibizumab and bevacizumab for treatment of neovascular age-related macular degeneration: two-year results. Ophthalmology 2012;119:1388-98.

8. Martin DF, Maguire MG, Ying GS, et al. Ranibizumab and bevacizumab for neovascular age-related macular degeneration. N Engl J Med 2011;364:1897-908.

9. Mitchell P, Annemans L, White R, et al. Cost effectiveness of treatments for wet age-related macular degeneration. Pharmacoeconomics 2011;29:107-31.

10. Yanagi Y, Aihara Y, Fukuda T, et al. [Cost-effectiveness of ranibizumab, photodynamic therapy and pegaptanib sodium in the treatment of neovascular age-related macular degeneration in Japanese]. Nihon Ganka Gakkai Zasshi 2011;115:825-31.

11. Patel JJ, Mendes MA, Bounthavong M, et al. Cost-utility analysis of bevacizumab versus ranibizumab in neovascular age-related macular degeneration using a Markov model. J Eval Clin Pract 2012;18:247-55.

12. Gower EW, Cassard SD, Bass EB, et al. A cost-effectiveness analysis of three treatments for age-related macular degeneration. Retina 2010;30:212-21.

13. Hernandez-Pastor LJ, Ortega A, Garcia-Layana A, et al. Cost-effectiveness of ranibizumab compared with pegaptanib in neovascular age-related macular degeneration. Graefes Arch Clin Exp Ophthalmol 2010;248:467-76.

14. Neubauer AS, Holz FG, Sauer S, et al. Cost-effectiveness of ranibizumab for the treatment of neovascular age-related macular degeneration in Germany: model analysis from the perspective of Germany's statutory health insurance system. Clin Ther 2010;32:1343-56.

15. Smiddy WE. Economic implications of current age-related macular degeneration treatments. Ophthalmology 2009;116:481-7.

16. Stein JD, Newman-Casey PA, Mrinalini T, et al. Cost-effectiveness of bevacizumab and ranibizumab for newly diagnosed neovascular macular degeneration. Ophthalmology 2014;121:936-45.

17. Fletcher EC, Lade RJ, Adewoyin T, et al. Computerized model of cost-utility analysis for treatment of age-related macular degeneration. Ophthalmology 2008;115:2192-8.

18. Raftery J, Clegg A, Jones J, et al. Ranibizumab (Lucentis) versus bevacizumab (Avastin): modelling cost effectiveness. $\mathrm{Br}$ Ophthalmol 2007;91:1244-6.

19. National Institute for Health and Care Excellence. Guide to the methods of technology appraisal 2013. Secondary Guide to the methods of technology appraisal 2013. 2013. http://www.nice.org.uk/ media/D45/1E/GuideToMethodsTechnologyAppraisal2013.pdf

20. Drummond MF, Sculpher MJ, Torrance GW, et al. Methods for the economic evaluation of health care programmes. 3rd edn. New York: Oxford University Press, 2005.

21. Dakin $\mathrm{H}$, Wordsworth $\mathrm{S}$. Cost-minimisation analysis versus cost-effectiveness analysis, revisited. Health Econ 2013;22:22-34

22. Briggs $\mathrm{AH}$, O'Brien BJ. The death of cost-minimization analysis? Health Econ 2001;10:179-84.

23. Jonsson B, Weinstein MC. Economic evaluation alongside multinational clinical trials. Study considerations for GUSTO Ilb. Int $J$ Technol Assess Health Care 1997;13:49-58.

24. Department of Health. PbR operational guidance and tariffs: 201112 tariff information spreadsheet. Secondary $\mathrm{PbR}$ operational guidance and tariffs: 2011-12 tariff information spreadsheet. 2011. http://www.dh.gov.uk/en/Publicationsandstatistics/Publications/ PublicationsPolicyAndGuidance/DH_124356

25. Department of Health. National Schedule of Reference Costs 201011 for NHS Trusts and PCTs combined. Secondary National Schedule of Reference Costs 2010-11 for NHS Trusts and PCTs combined. November 2011. http://www.dh.gov.uk/prod_consum_dh/ groups/dh_digitalassets/documents/digitalasset/dh 131148.xls

26. Curtis L. Unit Costs of Health and Social Care 2011. Pages 91 129-150, 177-213. Secondary Unit Costs of Health and Social Care 2011. Pages 91, 129-150, 177-213, 2011. http://www.pssru.ac.uk/ archive/pdf/uc/uc2011/uc2011.pdf

27. Dolan P. Modeling valuations for EuroQol health states. Med Care 1997;35:1095-108. 
28. White IR, Royston P, Wood AM. Multiple imputation using chained equations: issues and guidance for practice. Stat Med 2011;30:377-99.

29. Royston P. Multiple imputation of missing values: further update of ice, with an emphasis on interval censoring. Stata J 2007;7:445-64.

30. Hallstrom AP, Sullivan SD. On estimating costs for economic evaluation in failure time studies. Med Care 1998:36:433-6.

31. Gray A, Clarke P, Wolstenholme J, et al. Applied methods of cost-effectiveness analysis in health care. Oxford: Oxford University Press, 2011.

32. National Institute for Health and Clinical Excellence. Social value judgements: Principles for the development of NICE guidance. 2nd edn. Secondary Social value judgements: Principles for the development of NICE guidance. 2nd edn. 2008. http://www.nice.org uk/media/C18/30/SVJ2PUBLICATION2008.pdf

33. National Institute for Health and Clinical Excellence. Ranibizumab and pegaptanib for the treatment of age-related macular degeneration TA155: Costing template and report. Secondary Ranibizumab and pegaptanib for the treatment of age-related macular degeneration TA155: Costing template and report. August 2008. http://www.nice.org.uk/nicemedia/live/12057/41735/41735.xls

34. General Medical Council. Good practice in prescribing and managing medicines and devices. Secondary good practice in prescribing and managing medicines and devices. 2013. http://www. gmc-uk.org/static/documents/content/Prescribing_guidance.pdf

35. Curtis LH, Hammill BG, Schulman KA, et al. Risks of mortality, myocardial infarction, bleeding, and stroke associated with therapies for age-related macular degeneration. [Erratum appears in Arch Ophthalmol 2010;128:1623]. Arch Ophthalmol 2010;128:1273-9.

36. Bayer Plc. Summary of product characteristics for Eylea $40 \mathrm{mg} / \mathrm{mL}$ solution for injection in a vial Secondary Summary of product characteristics for Eylea $40 \mathrm{mg} / \mathrm{mL}$ solution for injection in a vial. 2013. http://www.medicines.org.uk/emc/medicine/27224/SPC/Eylea $+40 \mathrm{mg}+\mathrm{ml}+$ solution+for+injection+in+a+vial/ 